\title{
Lesión diafragmática durante la laparoscopia urológica transperitoneal*
}

\author{
Drs. OCTAVIO A. CASTILLO C. ${ }^{1,2,3}$, GONZALO VITAGLIANO ${ }^{1}$, IVAR VIDAL M. ${ }^{1}$
}

Unidad de Urología Clínica Indisa.

2 Facultad de Medicina, Universidad Andrés Bello.

3 Facultad de Medicina, Universidad de Chile.

Santiago, Chile.

\begin{abstract}
Diaphragmatic injury during transperitoneal urological laparoscopic surgery

Introduction: Capnothorax is a rare complication of urologic laparoscopy. However with the increasing use of this technique in a variety of urological procedures, this rare complication is a potential risk. Material and Methods: We analyzed a total of 786 urological procedures performed by transperitoneal laparoscopy in our center. All procedures were performed by the same surgeon: 213 adrenalectomy, 181 simple nephrectomies, 143 lymphadenectomies, 118 radical nephrectomies, 107 partial nephrectomies and 24 nephroureterectomy. Results: A total of 6 patients $(0.7 \%)$ present diaphragmatic lesions. The diaphragmatic repair was performed totally intracorporeal. One patient required the placement of a pleural drainage. No patient presented complications associated with diaphragmatic injury. Conclusion: Repair of diaphragmatic injury during transperitoneal laparoscopy can be performed successfully by this route. This technique is feasible, reproducible and reliable. This is the largest series reported by a single center.
\end{abstract}

Key words: Pneumothorax, diaphragm, intraoperative complications, laparoscopy.

\section{Resumen}

Introducción: El capnotórax es una complicación infrecuente de la laparoscopía urológica. No obstante, con el uso cada vez mayor de esta técnica en una gran variedad de procedimientos urológicos, esta infrecuente complicación se presenta como un riesgo potencial. Material y Métodos: Se analizaron un total de 786 procedimientos urológicos realizados en forma laparoscópica por vía transperitoneal en nuestro centro. Todos los procedimientos fueron realizados por el mismo cirujano: 213 adrenalectomías, 181 nefrectomías simples, 143 linfadenectomías, 118 nefrectomías radicales, 107 nefrectomías parciales y 24 nefroureterectomías. Resultados: Un total de 6 pacientes $(0,7 \%)$ presentaron lesiones diafragmáticas. La reparación diafragmática fue efectuada totalmente en forma intracorpórea. Un solo paciente requirió de la colocación de un drenaje pleural. Ningún paciente presentó complicaciones asociadas a la lesión diafragmática. Conclusión: La reparación de las lesiones diafragmáticas ocurridas durante la laparoscopía transperitoneal puede ser efectuada exitosamente por esta misma vía. Esta técnica es factible, reproducible y confiable. Esta es la serie más grande reportada por un solo centro.

Palabras clave: Pneumotórax, diafragma, complicación intraoperatoria, laparoscopía.

*Recibido el 28 de Julio de 2009 y aceptado para publicación el 10 de Diciembre de 2009.

Correspondencia: Dr. Octavio A. Castillo C.

Av. Apoquindo 3990, Of. 809. Santiago, Chile. Código postal: 7550112. Fax: (56-2) 2282524

E-mail: octaviocastillo@vtr.net 


\section{Introducción}

Las claras ventajas de la laparoscopía han popularizado este tipo de abordaje en la última década. No obstante, sólo en los últimos años, estas técnicas se han difundido en el ámbito urológico. La lesión diafragmática durante la laparoscopía urológica es una complicación muy infrecuente ${ }^{1}$. Pese a esto, la utilización cada vez mayor de esta vía en la resolución de patología urológica, hace que esta complicación permanezca como un riesgo potencial de este tipo de procedimiento. El capnotórax asociado a la cirugía laparoscópica es diferente del neumotórax común y requiere de un manejo específico.

Presentamos el manejo utilizado para la resolución de 6 casos de lesión diafragmática durante cirugía laparoscópica urológica.

\section{Material y Método}

Se revisaron los registros clínicos de 786 cirugías laparoscópicas transperitoneales. Todos los procedimientos fueron efectuados por un mismo cirujano: 213 adrenalectomías, 181 nefrectomías simples, 143 linfadenectomías, 118 nefrectomías radicales, 107 nefrectomías parciales y 24 nefroureterectomías.

Se encontraron un total de 6 lesiones diafragmáticas. La edad promedio de los pacientes fue de 53,2 años (rango 24 a 70). El IMC promedio fue de 28,15 (rango 20 a 48,9). Se analizaron los registros clínicos y quirúrgicos de estos pacientes. El tipo y modo de lesión diafragmática fue revisado, como también la técnica empleada para su resolución. El criterio utilizado para indicar la colocación del drenaje pleural fue de un capnotórax superior al $20 \%$, o la asociación a alteraciones hemodinámicas $\mathrm{y} / \mathrm{o}$ ventilatorias.

\section{Resultados}

Un total de 6 lesiones diafragmáticas fueron detectadas en nuestro análisis. Estas se presentaron durante 3 adrenalectomías, una nefrectomía parcial, una nefrectomía radical y una linfadenectomía retroperitoneal laparoscópica. En todos los casos las lesiones diafragmáticas fueron producidas en forma iatrogénica.

\section{Técnica de reparación}

Todas las lesiones diafragmáticas fueron reparadas en forma totalmente intracorpórea (Figura 1). En todos los casos el defecto se reparó con poliglactina 0 en aguja $\mathrm{CT}$ mientras se disminuía el pneumoperitoneo a $12 \mathrm{mmHg}$ y el anestesiólogo realizaba una hiperinsuflación forzada en el momento del cierre.

En la Tabla 1 se resumen los datos perioperatorios y resultados en los seis pacientes reportados.

\section{Discusión}

La lesión iatrogénica del diafragma durante los procedimientos laparoscópicos es verdaderamente infrecuente. Su incidencia no excede el $0,6 \%$ en las grandes series de nefrectomía y adrenalectomía laparoscópica ${ }^{2}$. Esta tan baja incidencia se explica por la clara separación que existe entre los riñones y el diafragma ${ }^{1}$. No obstante, la difusión masiva de la cirugía laparoscópica ha permitido que cada vez más cirujanos intenten realizar procedimientos de mayor complejidad. Esta práctica puede mantener o incluso aumentar la incidencia de lesiones diafragmáticas iatrogénicas. Es importante recalcar que esta serie refleja la experiencia personal de un cirujano autodidacta que ha superado la curva de aprendizaje.

En la mayoría de los casos de nuestra serie existían factores asociados que podrían haber facilitado la lesión diafragmática. Factores como obesidad mórbida, gran tamaño tumoral, enfermedad inflamatoria intestinal, cirugías previas y quimioterapia. Por otro lado, la cirugía adrenal en si misma es un factor que puede facilitar la ocurrencia de este tipo de complicación dado a la íntima relación entre la glándula suprarrenal y el diafragma.

La lesión diafragmática en la cirugía transperitoneal puede ser causada por la colocación incorrecta de un trócar o por el contacto directo de un bisturí monopolar o del bisturí armónico ${ }^{2}$. Cuando la retroperitoneoscopía es utilizada para la cirugía renal o adrenal, la colocación inadecuada de los trocares suele ser la responsable más frecuente de este tipo de lesión.

La lesión diafragmática puede ser diagnosticada mediante la visión directa mientras esta ocurre, o puede ser evidenciada indirectamente por cambios cardiorrespiratorios. El pneumotórax puede producir cambios auscultatorios, en la presión de fin de inspiración, en la presión sanguínea y en los gases arteriales ${ }^{4}$. Por otro lado, una lesión inadvertida puede evidenciarse por la aparición del signo de "floppy diaphragm" que se produce por abombamiento diafragmático al perderse la presión negativa en el momento del pneumotórax ${ }^{8}$. Este signo fue observado en 3 de los 6 pacientes de nuestra serie. Creemos que la participación del anestesiólogo es crucial durante el diagnóstico y reparación de estas lesiones ya que el capnotórax puede pasar desapercibido durante la cirugía y la alteración puede evidenciarse únicamente por alteraciones cardiopulmonares. 
LESIÓN DIAFRAGMÁTICA DURANTE LA LAPAROSCOPIA UROLÓGICA TRANSPERITONEAL

Tabla 1. Datos perioperatorios y resultados

\begin{tabular}{|c|c|c|c|c|c|c|c|c|c|}
\hline Caso & Edad & Sexo & IMC & Cirugía & $\begin{array}{c}\text { Tamaño } \\
\text { tumoral } \\
(\mathrm{cm})\end{array}$ & Reparación & $\begin{array}{c}\text { Tubo } \\
\text { Tx }\end{array}$ & $\begin{array}{l}\text { Cirugía } \\
\text { previa }\end{array}$ & $\begin{array}{c}\text { Patología } \\
\text { asociada }\end{array}$ \\
\hline 1 & 24 & $\mathrm{~F}$ & 21 & $\begin{array}{l}\text { Adrenalectomía } \\
\text { izquierda }\end{array}$ & 12 & Intracorpórea & Sí & No & $\begin{array}{l}\text { Colitis } \\
\text { ulcerosa }\end{array}$ \\
\hline 2 & 40 & $\mathrm{~F}$ & 24,5 & $\begin{array}{l}\text { Adrenalectomía } \\
\text { derecha }\end{array}$ & 7 & Intracorpórea & No & No & No \\
\hline 3 & 70 & $\mathrm{~F}$ & 48,9 & $\begin{array}{l}\text { Nefrectomía } \\
\text { parcial derecha }\end{array}$ & 3,5 & Intracorpórea & No & $\begin{array}{l}\text { Colecistecto- } \\
\text { mía }\end{array}$ & No \\
\hline 4 & 52 & M & 32 & $\begin{array}{l}\text { Nefrectomía } \\
\text { parcial izquierda }\end{array}$ & 5 & Intracorpórea & No & $\begin{array}{l}\text { Nefrectomía } \\
\text { parcial abierta }\end{array}$ & No \\
\hline 5 & 68 & M & 20 & $\begin{array}{l}\text { Linfadenectomía } \\
\text { retroperitoneal }\end{array}$ & 7 & Intracorpórea & No & $\begin{array}{l}\text { Nefroureterec- } \\
\text { tomía laparos- } \\
\text { cópica }\end{array}$ & $\begin{array}{l}\text { Quimio- } \\
\text { terapia } \\
\text { previa }\end{array}$ \\
\hline 6 & 65 & $\mathrm{~F}$ & 22,5 & $\begin{array}{l}\text { Adrenalectomía } \\
\text { izquierda }\end{array}$ & 5 & Intracorpórea & No & No & No \\
\hline
\end{tabular}

Para poder evitar las lesiones diafragmáticas se debe tener sumo cuidado a la hora de realizar disecciones y movilizaciones de las estructuras intraabdominales durante la exposición renal.

Es de importancia establecer el momento de la reparación diafragmática. Esta debe ser llevada a cabo de acuerdo con los parámetros cardiorrespiratorios del paciente y la factibilidad de la misma. En el caso donde la masa objeto de resección no permita el acceso directo a la lesión, la disección debe ser terminada antes de intentar la reparación.

No obstante, creemos que el defecto diafragmático debe ser reparado sin retraso en los casos en que esto sea factible. Este fue el caso en nuestra serie, donde todas las lesiones fueron rápidamente advertidas y reparadas sin retraso.

Nosotros utilizamos sutura interrumpida de poliglactina para la reparación de este tipo de lesión y creemos que esta técnica debe ser de primera elección ya que es reproducible y segura. Otros autores han presentado múltiples opciones para la reparación de las lesiones diafragmáticas. En un caso en particular, se utilizó una malla doble de polipropileno y poliglactina en una lesión de $1 \mathrm{~cm}$ producida durante una nefrectomía manoasistida. La malla fue fijada a la pared diafragmática utilizando una engrapadora laparoscópica con la ayuda de la mano del cirujano ${ }^{3}$. No obstante, muchos reportes muestran la factibilidad de la reparación mediante sutura intracorpórea ${ }^{2,4,5}$. Por otro lado, existe un reporte exitoso de cierre del defecto diafragmático sin la utilización de suturas ${ }^{7}$. Esto se logró utilizando una

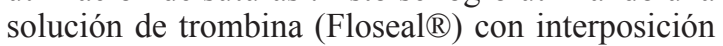

de epiplón sobre un defecto diafragmático de $1 \mathrm{~cm}$. No obstante, creemos que la experiencia es limitada y que esta técnica no ofrece la seguridad necesaria como para poder ser considerada como una opción.

Para que la reparación diafragmática sea efectiva, el dióxido de carbono debe ser evacuado previo al anudamiento de la sutura, tanto sea por medio de aspiración directa o mediante la administración de una inspiración forzada por parte del anestesiólogo.

Cuando el pneumotórax es mayor al $20 \%$, o se asocia a importantes cambios cardiopulmonares, la colocación de un tubo pleural se vuelve indispensa$b^{5}$. A su vez, las lesiones producidas por la colocación indebida de trocares o también la presencia de capnotórax residual requieren de pleurostomía. Por otro lado, es importante mencionar que el capnotórax puede resolverse en forma espontánea, permitiendo el manejo expectante en pacientes estables ${ }^{5}$.

En el primer caso de nuestra serie, importantes cambios ventilatorios evidenciaron el capnotórax, no obstante, el paciente permaneció hemodinámicamente estable. Creemos que la falta de experiencia en el manejo de esta eventualidad hizo que se colocara una pleurostomía en forma apresurada. En nuestra serie no se observaron lesiones producidas por la colocación de trocares. Esto se relaciona directamente con nuestra preferencia por la vía transperitoneal.

\section{Conclusiones}

Pese a ser muy infrecuente, la lesión diafragmática iatrogénica continua siendo una complicación potencial de la cirugía laparoscópica transperitoneal. 
Las características de los pacientes, el tamaño de las masas y la cirugía adrenal per se están asociados directamente con una mayor incidencia de este tipo de complicación. La reparación de estas lesiones debe ser realizada mediante sutura intracorpórea ya que este método es reproducible y seguro.

\section{Referencias}

1. Vallancien G, Cathelineau X, Baumert H, Doublet J, Gillonneau B. Complications of laparoscopic transperitoneal surgery in urology: review of 1.311 procedures at a single institution. J Urol 2002; 168: 23-26.

2. Del Pizzo J, Jacobs S, Bishoff J, Kavoussi L, Jarrett T. Pleural injury during laparoscopic renal surgery: early recognition and management. J Urol 2003; 169: 41-44.

3. González C, Batler R, Feldman M, Rubenstein J, Nadler R, Schoor R. Repair of a diaphragmatic injury during hand assisted laparoscopic nephrectomy using an onlay patch of polypropylene and polyglactin mesh. J Urol 2002; 167: 2512-2513.

4. Potter S, Kavoussi L, Jackman S. Management of diaphragmatic injury during laparoscopic nephrectomy. J Urol 2001; 165: 1203-1204.

5. Venkatesh R, Kibel A, Lee D, Reiiman J, Landman J. Rapid resolution of carbon dioxide pneumothorax (capno-thorax) resulting from diaphragmatic injury during laparoscopic nephrectomy. J Urol 2002; 167: 1387-1388.

6. Shanberg A, Zagnoev M, Clougherty T. Tension pneumothorax caused by the argon beam coagulator during laparoscopic partial nephrectomy. J Urol 2002; 168: 2162-2164.

7. Biiayani S, Grubb R, Andriole G. Use of gelatin matrix to rapidly repair diaphragmatic injury during laparoscopy. Urology 2002; 60: 514i-514ii.

8. Voyles C, Madden B. The "floppy diaphragm" sign with laparoscopic-associated pneumotorax. JSLS 1998; 2: 71-73. 